Acta Crystallographica Section E

Structure Reports

Online

ISSN 1600-5368

\section{2-[(3R,6R)-6-Methyl-2,5-dioxomorph- olin-3-yl]-N-(propan-2-yl)acetamide}

\section{De-dai Lu,* Hu Zhang, Juan Luo, Li-qiang Yang and Peng-xue Duan}

Key Laboratory of Polymer Materials of Gansu Province, Key Laboratory of EcoEnvironment-Related Polymer Materials, Ministry of Education, College of Chemistry and Chemical Engineering, Northwest Normal University, Lanzhou 730070, People's Republic of China

Correspondence e-mail: ludedai@126.com

Received 8 December 2011; accepted 22 February 2012

Key indicators: single-crystal X-ray study; $T=296 \mathrm{~K}$; mean $\sigma(\mathrm{C}-\mathrm{C})=0.004 \AA$; $R$ factor $=0.036 ; w R$ factor $=0.085 ;$ data-to-parameter ratio $=7.3$.

The molecular conformation of the title compound, $\mathrm{C}_{10} \mathrm{H}_{16} \mathrm{~N}_{2} \mathrm{O}_{4}$, is determined by an intramolecular $\mathrm{N}-\mathrm{H} \cdots \mathrm{O}$ hydrogen bond involving the morpholine $\mathrm{NH}$ group and the amide $\mathrm{O}$ atom. In the crystal, molecules are linked by $\mathrm{N}-$ $\mathrm{H}$.. O hydrogen bonds into chains along the $a$-axis direction.

\section{Related literature}

For the synthesis of polydepsipeptides, see: Feng et al. (2002); Hughes \& Sleebs (2005); In't Veld et al. (1992,1994); Jörres et al. (1998). For the synthesis of title compound, see: Wang \& Feng (1997).

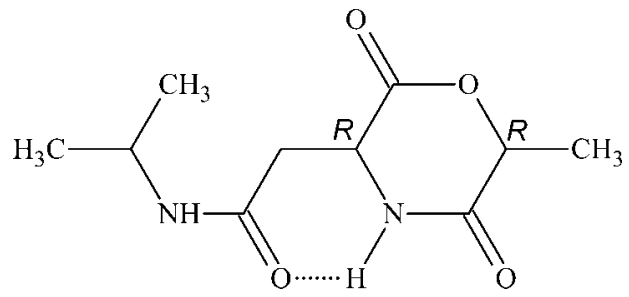

\section{Experimental}

Crystal data

\section{$\mathrm{C}_{10} \mathrm{H}_{16} \mathrm{~N}_{2} \mathrm{O}_{4}$}

$M_{r}=228.25$

Monoclinic, $P 2_{1}$
$=8.038(3) \mathrm{A}$

$b=5.678(2) \mathrm{A}$

$c=12.656(5) \AA$ $\beta=105.476(4)^{\circ}$

$V=556.6(3) \AA^{3}$

$Z=2$

Mo $K \alpha$ radiation

$\mu=0.11 \mathrm{~mm}^{-1}$

$T=296 \mathrm{~K}$

$0.28 \times 0.26 \times 0.24 \mathrm{~mm}$

Data collection

Bruker APEXII CCD

diffractometer

Absorption correction: multi-scan

(SADABS; Bruker, 2009)

$T_{\min }=0.971, T_{\max }=0.975$

Refinement

$R\left[F^{2}>2 \sigma\left(F^{2}\right)\right]=0.036$

$w R\left(F^{2}\right)=0.085$

$S=1.16$

1111 reflections

153 parameters

1 restraint

3796 measured reflections

1111 independent reflections

973 reflections with $I>2 \sigma(I)$

$R_{\text {int }}=0.026$

$\mathrm{H}$ atoms treated by a mixture of independent and constrained refinement

$\Delta \rho_{\max }=0.13 \mathrm{e} \AA^{-3}$

$\Delta \rho_{\min }=-0.13$ e $\AA^{-3}$

Table 1

Hydrogen-bond geometry $\left(\AA,^{\circ}\right)$.

\begin{tabular}{lllll}
\hline$D-\mathrm{H} \cdots A$ & $D-\mathrm{H}$ & $\mathrm{H} \cdots A$ & $D \cdots A$ & $D-\mathrm{H} \cdots A$ \\
\hline $\mathrm{N} 1-\mathrm{H} 1 \mathrm{~N} \cdots \mathrm{O} 4$ & $0.85(4)$ & $2.09(3)$ & $2.763(4)$ & $136(3)$ \\
$\mathrm{N} 2-\mathrm{H} 2 \mathrm{~N} \cdots \mathrm{O} 1^{\mathrm{i}}$ & $0.82(3)$ & $2.11(3)$ & $2.926(3)$ & $170(3)$ \\
\hline
\end{tabular}

Symmetry code: (i) $x+1, y, z$.

Data collection: APEX2 (Bruker, 2009); cell refinement: SAINT (Bruker, 2009); data reduction: $S A I N T$; $\operatorname{program}(\mathrm{s})$ used to solve structure: SHELXS97 (Sheldrick, 2008); program(s) used to refine structure: SHELXL97 (Sheldrick, 2008); molecular graphics: SHELXTL (Sheldrick, 2008); software used to prepare material for publication: SHELXTL.

We are thankful for the financial support of this work by the National Natural Science Foundation of China (grant No. 51103118).

Supplementary data and figures for this paper are available from the IUCr electronic archives (Reference: YK2036).

\title{
References
}

Bruker (2009). APEX2, SAINT and SADABS. Bruker AXS Inc., Madison, Wisconsin, USA.

Feng, Y.-K., Klee, D. \& Höcker, H. (2002). Macromol. Chem. Phys. 203, 819824.

Hughes, A. B. \& Sleebs, M. M. (2005). J. Org. Chem. 70, 3079-3088.

In't Veld, P. J. A., Dijkstra, P. J. \& Feijen, J. (1992). Macromol. Chem. Phys. 193, 2713-2730.

In’t Veld, P. J. A., Shen, Z. R., Takens, G. A. J., Dijkstra, P. J. \& Feijen, J. (1994). J. Polym. Sci. Part A Polym. Chem. 32, 1063-1069.

Jörres, V., Keul, H. \& Höcker, H. (1998). Macromol. Chem. Phys. 199, 825-833.

Sheldrick, G. M. (2008). Acta Cryst. A64, 112-122.

Wang, D. \& Feng, X.-D. (1997). Macromolecules, 30, 5688-5692. 


\section{supporting information}

Acta Cryst. (2012). E68, o896 [doi:10.1107/S1600536812007945]

\section{2-[(3R,6R)-6-Methyl-2,5-dioxomorpholin-3-yl]-N-(propan-2-yl)acetamide}

\section{De-dai Lu, Hu Zhang, Juan Luo, Li-qiang Yang and Peng-xue Duan}

\section{S1. Comment}

Polydepsipeptides, copolymers of $\alpha$-hydroxy acids and $\alpha$-amino acids, are the most important representatives of biodegradable polyesteramides. Morpholine-2,5-dione derivatives are a series of monomers which were used to synthesize polydepsipeptides via ring-opening polymerization. (In't Veld et al., 1992,1994). In recent years, all kinds of functional groups are introduced into these monomers in order to synthesize functional polydepsipeptides (Feng et al., 2002; Jörres et al., 1998; Hughes \& Sleebs, 2005). In our current research, related to this topic, we have designed and synthesized the title compound, which includes the isopropyl amide functional group (Fig. 1). In the crystal structure of the title compound, $\mathrm{C}_{10} \mathrm{H}_{16} \mathrm{~N}_{2} \mathrm{O}_{4}$, there are two kinds of hydrogen bonds, one is intramolecular $\mathrm{N}-\mathrm{H} \cdots \mathrm{O}[\mathrm{H} \cdots \mathrm{O}=2.09$ (3) $\AA]$ hydrogen bond, the other is intermolecular $\mathrm{N}-\mathrm{H} \cdots \mathrm{O}[\mathrm{H} \cdots \mathrm{O}=2.11$ (3) $\AA]$ hydrogen bond which links molecules into the one-dimensional chains along the $a$-axis direction (Fig. 2).

\section{S2. Experimental}

All reagents used in the syntheses were of analytical grade and used without further purification. The title compound was prepared according to the literature method (Wang \& Feng, 1997). Single crystals were grown from ethyl acetate solution by slow evaporation at room temperature.

\section{S3. Refinement}

All $\mathrm{H}$ atoms were placed in calculated positions, with $\mathrm{C}-\mathrm{H}$ distances ranging from 0.96 to $0.98 \AA$, and $\mathrm{N}-\mathrm{H}$ distances ranging from 0.82 to $0.85 \AA$. They were refined in the riding-model approximation, with $U_{\text {iso }}(\mathrm{H})=1.2 U_{\text {eq }}($ carrier $\mathrm{C}, \mathrm{N})$ or $U_{\text {iso }}(\mathrm{H})=1.5 U_{\mathrm{eq}}(\mathrm{C}$ of methyl group $)$. 


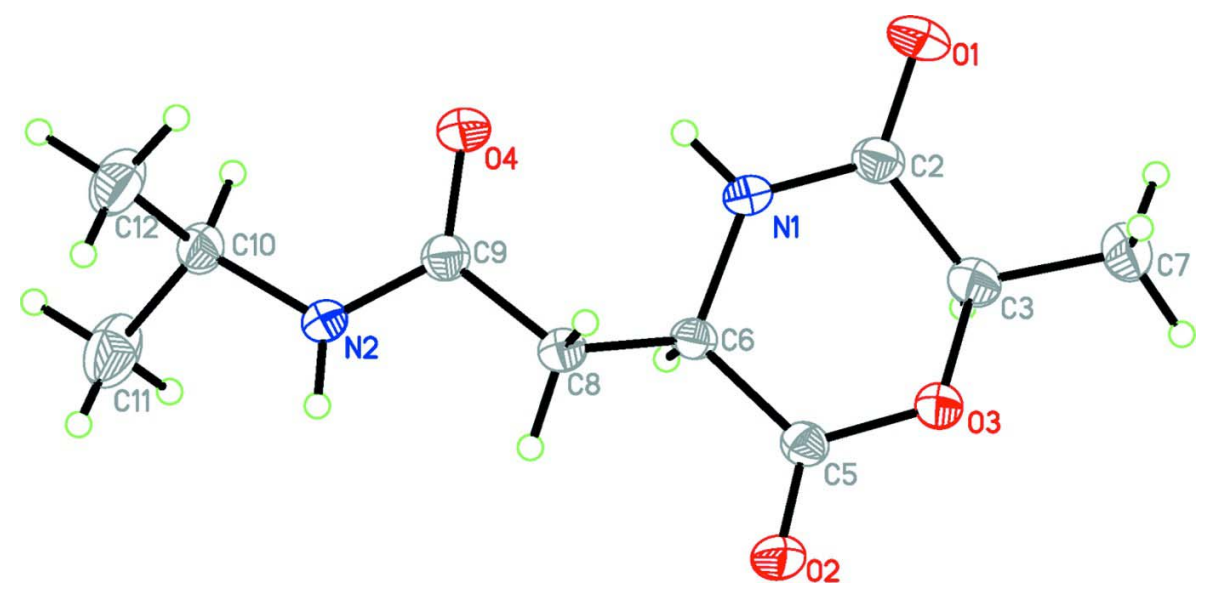

\section{Figure 1}

Molecular structure of the title compound showing the atom labelling scheme. Displacement ellipsoids are drawn at the $30 \%$ probability level.

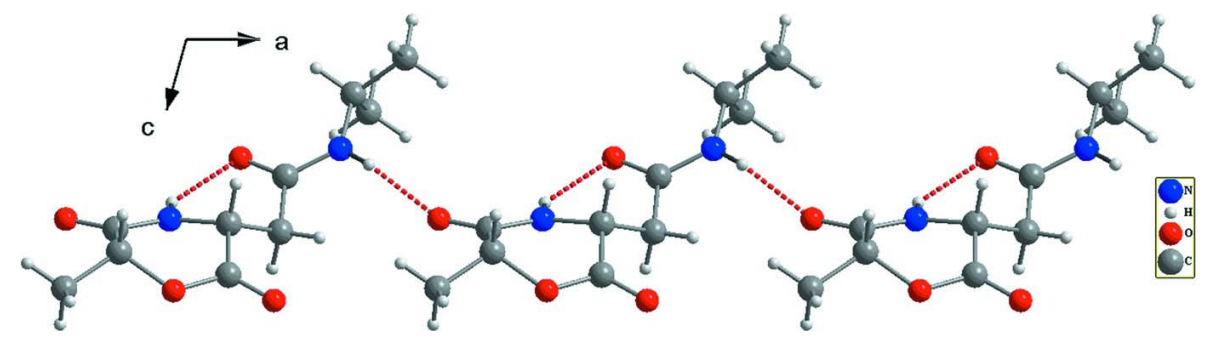

\section{Figure 2}

The hydrogen-bonded chain in the structure of title compound.

\section{2-[(3R,6R)-6-methyl-2,5-dioxomorpholin-3-yl]- N-(propan-2-yl)acetamide}

\section{Crystal data}

$\mathrm{C}_{10} \mathrm{H}_{16} \mathrm{~N}_{2} \mathrm{O}_{4}$

$M_{r}=228.25$

Monoclinic, $P 2_{1}$

Hall symbol: $\mathrm{P} 2 \mathrm{yb}$

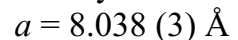

$b=5.678(2) \AA$

$c=12.656(5) \AA$

$\beta=105.476(4)^{\circ}$

$V=556.6(3) \AA^{3}$

$Z=2$

\section{Data collection}

\section{Bruker APEXII CCD}

diffractometer

Radiation source: fine-focus sealed tube

Graphite monochromator

$\varphi$ and $\omega$ scans

Absorption correction: multi-scan

(SADABS; Bruker, 2009)

$T_{\min }=0.971, T_{\max }=0.975$
$F(000)=244$

$D_{\mathrm{x}}=1.362 \mathrm{Mg} \mathrm{m}^{-3}$

Mo $K \alpha$ radiation, $\lambda=0.71073 \AA$

Cell parameters from 2019 reflections

$\theta=2.6-28.4^{\circ}$

$\mu=0.11 \mathrm{~mm}^{-1}$

$T=296 \mathrm{~K}$

Block, colourless

$0.28 \times 0.26 \times 0.24 \mathrm{~mm}$

3796 measured reflections

1111 independent reflections

973 reflections with $I>2 \sigma(I)$

$R_{\text {int }}=0.026$

$\theta_{\max }=25.5^{\circ}, \theta_{\min }=2.6^{\circ}$

$h=-9 \rightarrow 8$

$k=-6 \rightarrow 6$

$l=-15 \rightarrow 15$ 


\section{Refinement}

Refinement on $F^{2}$

Least-squares matrix: full

$R\left[F^{2}>2 \sigma\left(F^{2}\right)\right]=0.036$

$w R\left(F^{2}\right)=0.085$

$S=1.16$

1111 reflections

153 parameters

1 restraint

Primary atom site location: structure-invariant direct methods
Secondary atom site location: difference Fourier map

Hydrogen site location: inferred from neighbouring sites

$\mathrm{H}$ atoms treated by a mixture of independent and constrained refinement

$w=1 /\left[\sigma^{2}\left(F_{\mathrm{o}}^{2}\right)+(0.0381 P)^{2}+0.0663 P\right]$ where $P=\left(F_{\mathrm{o}}{ }^{2}+2 F_{\mathrm{c}}{ }^{2}\right) / 3$

$(\Delta / \sigma)_{\max }<0.001$

$\Delta \rho_{\max }=0.13$ e $\AA^{-3}$

$\Delta \rho_{\min }=-0.13$ e $\AA^{-3}$

\section{Special details}

Geometry. All s.u.'s (except the s.u. in the dihedral angle between two 1.s. planes) are estimated using the full covariance matrix. The cell s.u.'s are taken into account individually in the estimation of s.u.'s in distances, angles and torsion angles; correlations between s.u.'s in cell parameters are only used when they are defined by crystal symmetry. An approximate (isotropic) treatment of cell s.u.'s is used for estimating s.u.'s involving l.s. planes.

Refinement. Refinement of $F^{2}$ against ALL reflections. The weighted $R$-factor $w R$ and goodness of fit $S$ are based on $F^{2}$, conventional $R$-factors $R$ are based on $F$, with $F$ set to zero for negative $F^{2}$. The threshold expression of $F^{2}>\sigma\left(F^{2}\right)$ is used only for calculating $R$-factors(gt) $e t c$. and is not relevant to the choice of reflections for refinement. $R$-factors based on $F^{2}$ are statistically about twice as large as those based on $F$, and $R$ - factors based on ALL data will be even larger.

Fractional atomic coordinates and isotropic or equivalent isotropic displacement parameters $\left(\AA^{2}\right)$

\begin{tabular}{lllll}
\hline & $x$ & $y$ & $z$ & $U_{\text {iso }} * / U_{\text {eq }}$ \\
\hline N1 & $0.3090(3)$ & $0.0143(5)$ & $0.7877(2)$ & $0.0396(6)$ \\
H1N & $0.303(3)$ & $-0.121(7)$ & $0.759(2)$ & $0.041(9)^{*}$ \\
O1 & $0.0310(2)$ & $0.0204(5)$ & $0.78753(18)$ & $0.0587(6)$ \\
C2 & $0.1717(3)$ & $0.1165(6)$ & $0.8051(2)$ & $0.0395(7)$ \\
N2 & $0.7140(3)$ & $-0.2115(4)$ & $0.66322(19)$ & $0.0384(6)$ \\
H2N & $0.803(4)$ & $-0.138(6)$ & $0.691(2)$ & $0.041(9)^{*}$ \\
O2 & $0.6493(2)$ & $0.3811(4)$ & $0.93137(16)$ & $0.0480(6)$ \\
C3 & $0.1981(3)$ & $0.3664(6)$ & $0.8450(2)$ & $0.0425(7)$ \\
H3A & 0.1803 & 0.4699 & 0.7810 & $0.051^{*}$ \\
O3 & $0.3725(2)$ & $0.4038(4)$ & $0.91393(15)$ & $0.0426(5)$ \\
O4 & $0.4538(2)$ & $-0.3182(4)$ & $0.67977(16)$ & $0.0500(6)$ \\
C5 & $0.5079(3)$ & $0.3148(5)$ & $0.8859(2)$ & $0.0343(7)$ \\
C6 & $0.4708(3)$ & $0.1354(5)$ & $0.7952(2)$ & $0.0310(6)$ \\
H6A & 0.4607 & 0.2184 & 0.7259 & $0.037^{*}$ \\
C7 & $0.0782(4)$ & $0.4419(7)$ & $0.9102(3)$ & $0.0641(11)$ \\
H7A & 0.1023 & 0.6023 & 0.9332 & $0.096^{*}$ \\
H7B & 0.0939 & 0.3426 & 0.9735 & $0.096^{*}$ \\
H7C & -0.0388 & 0.4295 & 0.8659 & $0.096^{*}$ \\
C8 & $0.6152(3)$ & $-0.0437(5)$ & $0.8098(2)$ & $0.0351(7)$ \\
H8A & 0.7244 & 0.0380 & 0.8203 & $0.042^{*}$ \\
H8B & 0.6210 & -0.1370 & 0.8749 & $0.042^{*}$ \\
C9 & $0.5878(3)$ & $-0.2038(5)$ & $0.7122(2)$ & $0.0355(7)$ \\
C10 & $0.7090(3)$ & $-0.3549(6)$ & $0.5672(2)$ & $0.0409(7)$ \\
H10A & 0.5898 & -0.3578 & 0.5211 & $0.049^{*}$
\end{tabular}




$\begin{array}{lllll}\text { C11 } & 0.8204(5) & -0.2421(7) & 0.5025(3) & 0.0704(11) \\ \text { H11A } & 0.7822 & -0.0836 & 0.4839 & 0.106^{*} \\ \text { H11B } & 0.8118 & -0.3306 & 0.4366 & 0.106^{*} \\ \text { H11C } & 0.9384 & -0.2403 & 0.5458 & 0.106^{*} \\ \text { C12 } & 0.7629(5) & -0.6010(7) & 0.5983(3) & 0.0634(10) \\ \text { H12A } & 0.6895 & -0.6672 & 0.6392 & 0.095^{*} \\ \text { H12B } & 0.8803 & -0.6023 & 0.6424 & 0.095^{*} \\ \text { H12C } & 0.7538 & -0.6927 & 0.5332 & 0.095^{*}\end{array}$

Atomic displacement parameters $\left(\AA^{2}\right)$

\begin{tabular}{lllllll}
\hline & $U^{11}$ & $U^{22}$ & $U^{33}$ & $U^{12}$ & $U^{13}$ & $U^{23}$ \\
\hline $\mathrm{N} 1$ & $0.0359(13)$ & $0.0329(15)$ & $0.0509(16)$ & $-0.0098(12)$ & $0.0133(11)$ & $-0.0121(14)$ \\
O1 & $0.0304(11)$ & $0.0629(16)$ & $0.0810(15)$ & $-0.0155(11)$ & $0.0121(10)$ & $-0.0133(14)$ \\
C2 & $0.0326(15)$ & $0.0445(19)$ & $0.0402(15)$ & $-0.0056(14)$ & $0.0077(12)$ & $-0.0024(14)$ \\
N2 & $0.0345(13)$ & $0.0397(15)$ & $0.0424(14)$ & $-0.0085(12)$ & $0.0127(11)$ & $-0.0152(12)$ \\
O2 & $0.0342(10)$ & $0.0475(13)$ & $0.0588(12)$ & $-0.0081(11)$ & $0.0064(9)$ & $-0.0169(11)$ \\
C3 & $0.0330(14)$ & $0.0476(19)$ & $0.0453(17)$ & $-0.0025(15)$ & $0.0073(12)$ & $-0.0029(15)$ \\
O3 & $0.0322(9)$ & $0.0479(13)$ & $0.0481(11)$ & $-0.0059(10)$ & $0.0115(8)$ & $-0.0154(10)$ \\
O4 & $0.0389(11)$ & $0.0542(15)$ & $0.0574(13)$ & $-0.0152(11)$ & $0.0138(9)$ & $-0.0229(12)$ \\
C5 & $0.0346(15)$ & $0.0343(18)$ & $0.0359(14)$ & $-0.0045(13)$ & $0.0125(11)$ & $0.0009(13)$ \\
C6 & $0.0299(13)$ & $0.0330(16)$ & $0.0305(14)$ & $-0.0062(12)$ & $0.0088(10)$ & $-0.0015(13)$ \\
C7 & $0.0410(17)$ & $0.077(3)$ & $0.077(2)$ & $-0.0029(19)$ & $0.0215(15)$ & $-0.028(2)$ \\
C8 & $0.0330(14)$ & $0.0361(18)$ & $0.0351(14)$ & $-0.0045(13)$ & $0.0072(11)$ & $-0.0050(13)$ \\
C9 & $0.0340(14)$ & $0.0340(17)$ & $0.0375(15)$ & $-0.0004(13)$ & $0.0077(11)$ & $-0.0011(14)$ \\
C10 & $0.0370(15)$ & $0.0457(19)$ & $0.0394(16)$ & $0.0007(15)$ & $0.0091(12)$ & $-0.0102(15)$ \\
C11 & $0.112(3)$ & $0.051(2)$ & $0.063(2)$ & $-0.001(2)$ & $0.050(2)$ & $-0.003(2)$ \\
C12 & $0.091(3)$ & $0.039(2)$ & $0.069(2)$ & $0.002(2)$ & $0.035(2)$ & $-0.0075(19)$ \\
& & & & & & \\
\hline
\end{tabular}

Geometric parameters $\left(\AA,{ }^{\circ}\right)$

\begin{tabular}{llll}
\hline $\mathrm{N} 1-\mathrm{C} 2$ & $1.317(4)$ & $\mathrm{C} 6-\mathrm{H} 6 \mathrm{~A}$ & 0.9800 \\
$\mathrm{~N} 1-\mathrm{C} 6$ & $1.452(3)$ & $\mathrm{C} 7-\mathrm{H} 7 \mathrm{~A}$ & 0.9600 \\
$\mathrm{~N} 1-\mathrm{H} 1 \mathrm{~N}$ & $0.85(4)$ & $\mathrm{C} 7-\mathrm{H} 7 \mathrm{~B}$ & 0.9600 \\
$\mathrm{O} 1-\mathrm{C} 2$ & $1.222(3)$ & $\mathrm{C} 7-\mathrm{H} 7 \mathrm{C}$ & 0.9600 \\
$\mathrm{C} 2-\mathrm{C} 3$ & $1.502(5)$ & $\mathrm{C} 8-\mathrm{C} 9$ & $1.502(4)$ \\
$\mathrm{N} 2-\mathrm{C} 9$ & $1.323(3)$ & $\mathrm{C} 8-\mathrm{H} 8 \mathrm{~A}$ & 0.9700 \\
$\mathrm{~N} 2-\mathrm{C} 10$ & $1.454(4)$ & $\mathrm{C} 8-\mathrm{H} 8 \mathrm{~B}$ & 0.9700 \\
$\mathrm{~N} 2-\mathrm{H} 2 \mathrm{~N}$ & $0.82(3)$ & $\mathrm{C} 10-\mathrm{C} 12$ & $1.485(5)$ \\
$\mathrm{O} 2-\mathrm{C} 5$ & $1.190(3)$ & $\mathrm{C} 10-\mathrm{C} 11$ & $1.508(4)$ \\
$\mathrm{C} 3-\mathrm{O} 3$ & $1.456(3)$ & $\mathrm{C} 10-\mathrm{H} 10 \mathrm{~A}$ & 0.9800 \\
$\mathrm{C} 3-\mathrm{C} 7$ & $1.489(4)$ & $\mathrm{C} 11-\mathrm{H} 11 \mathrm{~A}$ & 0.9600 \\
$\mathrm{C} 3-\mathrm{H} 3 \mathrm{~A}$ & 0.9800 & $\mathrm{C} 11-\mathrm{H} 11 \mathrm{~B}$ & 0.9600 \\
$\mathrm{O} 3-\mathrm{C} 5$ & $1.331(3)$ & $\mathrm{C} 11-\mathrm{H} 11 \mathrm{C}$ & 0.9600 \\
$\mathrm{O} 4-\mathrm{C} 9$ & $1.231(3)$ & $\mathrm{C} 12-\mathrm{H} 12 \mathrm{~A}$ & 0.9600 \\
$\mathrm{C} 5-\mathrm{C} 6$ & $1.504(4)$ & $\mathrm{C} 12-\mathrm{H} 12 \mathrm{~B}$ & 0.9600 \\
$\mathrm{C} 6-\mathrm{C} 8$ & $1.517(4)$ & $\mathrm{C} 12-\mathrm{H} 12 \mathrm{C}$ & 0.9600
\end{tabular}




\begin{tabular}{|c|c|c|c|}
\hline $\mathrm{C} 2-\mathrm{N} 1-\mathrm{C} 6$ & $123.8(3)$ & $\mathrm{H} 7 \mathrm{~A}-\mathrm{C} 7-\mathrm{H} 7 \mathrm{C}$ & 109.5 \\
\hline $\mathrm{C} 2-\mathrm{N} 1-\mathrm{H} 1 \mathrm{~N}$ & $121.1(19)$ & $\mathrm{H} 7 \mathrm{~B}-\mathrm{C} 7-\mathrm{H} 7 \mathrm{C}$ & 109.5 \\
\hline $\mathrm{C} 6-\mathrm{N} 1-\mathrm{H} 1 \mathrm{~N}$ & $114.0(19)$ & $\mathrm{C} 9-\mathrm{C} 8-\mathrm{C} 6$ & $111.5(2)$ \\
\hline $\mathrm{O} 1-\mathrm{C} 2-\mathrm{N} 1$ & $123.3(3)$ & $\mathrm{C} 9-\mathrm{C} 8-\mathrm{H} 8 \mathrm{~A}$ & 109.3 \\
\hline $\mathrm{O} 1-\mathrm{C} 2-\mathrm{C} 3$ & $121.6(3)$ & $\mathrm{C} 6-\mathrm{C} 8-\mathrm{H} 8 \mathrm{~A}$ & 109.3 \\
\hline $\mathrm{N} 1-\mathrm{C} 2-\mathrm{C} 3$ & $115.0(3)$ & $\mathrm{C} 9-\mathrm{C} 8-\mathrm{H} 8 \mathrm{~B}$ & 109.3 \\
\hline $\mathrm{C} 9-\mathrm{N} 2-\mathrm{C} 10$ & $123.8(3)$ & $\mathrm{C} 6-\mathrm{C} 8-\mathrm{H} 8 \mathrm{~B}$ & 109.3 \\
\hline $\mathrm{C} 9-\mathrm{N} 2-\mathrm{H} 2 \mathrm{~N}$ & $118(2)$ & $\mathrm{H} 8 \mathrm{~A}-\mathrm{C} 8-\mathrm{H} 8 \mathrm{~B}$ & 108.0 \\
\hline $\mathrm{C} 10-\mathrm{N} 2-\mathrm{H} 2 \mathrm{~N}$ & $118(2)$ & $\mathrm{O} 4-\mathrm{C} 9-\mathrm{N} 2$ & $122.6(3)$ \\
\hline $\mathrm{O} 3-\mathrm{C} 3-\mathrm{C} 7$ & $106.7(2)$ & $\mathrm{O} 4-\mathrm{C} 9-\mathrm{C} 8$ & $121.1(2)$ \\
\hline $\mathrm{O} 3-\mathrm{C} 3-\mathrm{C} 2$ & $111.5(3)$ & $\mathrm{N} 2-\mathrm{C} 9-\mathrm{C} 8$ & $116.3(2)$ \\
\hline $\mathrm{C} 7-\mathrm{C} 3-\mathrm{C} 2$ & $113.8(3)$ & $\mathrm{N} 2-\mathrm{C} 10-\mathrm{C} 12$ & $111.4(3)$ \\
\hline $\mathrm{O} 3-\mathrm{C} 3-\mathrm{H} 3 \mathrm{~A}$ & 108.2 & $\mathrm{~N} 2-\mathrm{C} 10-\mathrm{C} 11$ & $109.1(3)$ \\
\hline $\mathrm{C} 7-\mathrm{C} 3-\mathrm{H} 3 \mathrm{~A}$ & 108.2 & $\mathrm{C} 12-\mathrm{C} 10-\mathrm{C} 11$ & $111.7(3)$ \\
\hline $\mathrm{C} 2-\mathrm{C} 3-\mathrm{H} 3 \mathrm{~A}$ & 108.2 & $\mathrm{~N} 2-\mathrm{C} 10-\mathrm{H} 10 \mathrm{~A}$ & 108.2 \\
\hline $\mathrm{C} 5-\mathrm{O} 3-\mathrm{C} 3$ & $120.7(2)$ & $\mathrm{C} 12-\mathrm{C} 10-\mathrm{H} 10 \mathrm{~A}$ & 108.2 \\
\hline $\mathrm{O} 2-\mathrm{C} 5-\mathrm{O} 3$ & $119.6(3)$ & $\mathrm{C} 11-\mathrm{C} 10-\mathrm{H} 10 \mathrm{~A}$ & 108.2 \\
\hline $\mathrm{O} 2-\mathrm{C} 5-\mathrm{C} 6$ & $123.5(2)$ & $\mathrm{C} 10-\mathrm{C} 11-\mathrm{H} 11 \mathrm{~A}$ & 109.5 \\
\hline $\mathrm{O} 3-\mathrm{C} 5-\mathrm{C} 6$ & $116.8(2)$ & $\mathrm{C} 10-\mathrm{C} 11-\mathrm{H} 11 \mathrm{~B}$ & 109.5 \\
\hline $\mathrm{N} 1-\mathrm{C} 6-\mathrm{C} 5$ & $111.3(2)$ & $\mathrm{H} 11 \mathrm{~A}-\mathrm{C} 11-\mathrm{H} 11 \mathrm{~B}$ & 109.5 \\
\hline $\mathrm{N} 1-\mathrm{C} 6-\mathrm{C} 8$ & $109.5(2)$ & $\mathrm{C} 10-\mathrm{C} 11-\mathrm{H} 11 \mathrm{C}$ & 109.5 \\
\hline $\mathrm{C} 5-\mathrm{C} 6-\mathrm{C} 8$ & $111.9(2)$ & $\mathrm{H} 11 \mathrm{~A}-\mathrm{C} 11-\mathrm{H} 11 \mathrm{C}$ & 109.5 \\
\hline $\mathrm{N} 1-\mathrm{C} 6-\mathrm{H} 6 \mathrm{~A}$ & 108.0 & $\mathrm{H} 11 \mathrm{~B}-\mathrm{C} 11-\mathrm{H} 11 \mathrm{C}$ & 109.5 \\
\hline $\mathrm{C} 5-\mathrm{C} 6-\mathrm{H} 6 \mathrm{~A}$ & 108.0 & $\mathrm{C} 10-\mathrm{C} 12-\mathrm{H} 12 \mathrm{~A}$ & 109.5 \\
\hline $\mathrm{C} 8-\mathrm{C} 6-\mathrm{H} 6 \mathrm{~A}$ & 108.0 & $\mathrm{C} 10-\mathrm{C} 12-\mathrm{H} 12 \mathrm{~B}$ & 109.5 \\
\hline $\mathrm{C} 3-\mathrm{C} 7-\mathrm{H} 7 \mathrm{~A}$ & 109.5 & $\mathrm{H} 12 \mathrm{~A}-\mathrm{C} 12-\mathrm{H} 12 \mathrm{~B}$ & 109.5 \\
\hline $\mathrm{C} 3-\mathrm{C} 7-\mathrm{H} 7 \mathrm{~B}$ & 109.5 & $\mathrm{C} 10-\mathrm{C} 12-\mathrm{H} 12 \mathrm{C}$ & 109.5 \\
\hline $\mathrm{H} 7 \mathrm{~A}-\mathrm{C} 7-\mathrm{H} 7 \mathrm{~B}$ & 109.5 & $\mathrm{H} 12 \mathrm{~A}-\mathrm{C} 12-\mathrm{H} 12 \mathrm{C}$ & 109.5 \\
\hline $\mathrm{C} 3-\mathrm{C} 7-\mathrm{H} 7 \mathrm{C}$ & 109.5 & $\mathrm{H} 12 \mathrm{~B}-\mathrm{C} 12-\mathrm{H} 12 \mathrm{C}$ & 109.5 \\
\hline $\mathrm{C} 6-\mathrm{N} 1-\mathrm{C} 2-\mathrm{O} 1$ & $172.1(3)$ & $\mathrm{O} 2-\mathrm{C} 5-\mathrm{C} 6-\mathrm{N} 1$ & $155.1(3)$ \\
\hline $\mathrm{C} 6-\mathrm{N} 1-\mathrm{C} 2-\mathrm{C} 3$ & $-5.8(4)$ & $\mathrm{O} 3-\mathrm{C} 5-\mathrm{C} 6-\mathrm{N} 1$ & $-25.9(3)$ \\
\hline $\mathrm{O} 1-\mathrm{C} 2-\mathrm{C} 3-\mathrm{O} 3$ & $149.0(3)$ & $\mathrm{O} 2-\mathrm{C} 5-\mathrm{C} 6-\mathrm{C} 8$ & $32.4(4)$ \\
\hline $\mathrm{N} 1-\mathrm{C} 2-\mathrm{C} 3-\mathrm{O} 3$ & $-33.1(3)$ & $\mathrm{O} 3-\mathrm{C} 5-\mathrm{C} 6-\mathrm{C} 8$ & $-148.7(2)$ \\
\hline $\mathrm{O} 1-\mathrm{C} 2-\mathrm{C} 3-\mathrm{C} 7$ & $28.2(4)$ & $\mathrm{N} 1-\mathrm{C} 6-\mathrm{C} 8-\mathrm{C} 9$ & $63.2(3)$ \\
\hline $\mathrm{N} 1-\mathrm{C} 2-\mathrm{C} 3-\mathrm{C} 7$ & $-153.9(3)$ & $\mathrm{C} 5-\mathrm{C} 6-\mathrm{C} 8-\mathrm{C} 9$ & $-173.0(2)$ \\
\hline $\mathrm{C} 7-\mathrm{C} 3-\mathrm{O} 3-\mathrm{C} 5$ & $168.1(3)$ & $\mathrm{C} 10-\mathrm{N} 2-\mathrm{C} 9-\mathrm{O} 4$ & $-0.3(4)$ \\
\hline $\mathrm{C} 2-\mathrm{C} 3-\mathrm{O} 3-\mathrm{C} 5$ & $43.3(4)$ & $\mathrm{C} 10-\mathrm{N} 2-\mathrm{C} 9-\mathrm{C} 8$ & $-179.4(3)$ \\
\hline $\mathrm{C} 3-\mathrm{O} 3-\mathrm{C} 5-\mathrm{O} 2$ & $166.4(3)$ & $\mathrm{C} 6-\mathrm{C} 8-\mathrm{C} 9-\mathrm{O} 4$ & $-55.6(3)$ \\
\hline $\mathrm{C} 3-\mathrm{O} 3-\mathrm{C} 5-\mathrm{C} 6$ & $-12.6(4)$ & $\mathrm{C} 6-\mathrm{C} 8-\mathrm{C} 9-\mathrm{N} 2$ & $123.5(3)$ \\
\hline $\mathrm{C} 2-\mathrm{N} 1-\mathrm{C} 6-\mathrm{C} 5$ & $36.3(4)$ & $\mathrm{C} 9-\mathrm{N} 2-\mathrm{C} 10-\mathrm{C} 12$ & $-83.3(3)$ \\
\hline $\mathrm{C} 2-\mathrm{N} 1-\mathrm{C} 6-\mathrm{C} 8$ & $160.4(2)$ & $\mathrm{C} 9-\mathrm{N} 2-\mathrm{C} 10-\mathrm{C} 11$ & $152.9(3)$ \\
\hline
\end{tabular}

Hydrogen-bond geometry $\left(A,{ }^{\circ}\right)$

\begin{tabular}{lllll}
\hline$D-\mathrm{H} \cdots A$ & $D-\mathrm{H}$ & $\mathrm{H} \cdots A$ & $D \cdots A$ & $D-\mathrm{H} \cdots A$ \\
\hline $\mathrm{N} 1-\mathrm{H} 1 N \cdots \mathrm{O} 4$ & $0.85(4)$ & $2.09(3)$ & $2.763(4)$ & $136(3)$
\end{tabular}


$\mathrm{N} 2-\mathrm{H} 2 N \cdots \mathrm{O} 1^{\mathrm{i}}$

0.82 (3)

Symmetry code: (i) $x+1, y, z$.
$2.11(3)$

$2.926(3)$

$170(3)$ 